\title{
Exploring the Complexities of Postpartum Sexual Health
}

\author{
Deirdre O’Malley ${ }^{1}\left[\right.$ Agnes Higgins $^{1} \cdot$ Valerie Smith $^{1}$
}

Accepted: 17 September 2021 / Published online: 5 October 2021

(c) The Author(s) 2021, corrected publication 2022

\begin{abstract}
Purpose of Review This paper explores the complexities of postpartum sexual health. It answers the question on what should be considered normal sexual health after birth and what should be considered abnormal.

Recent Findings Many women experience physical sexual health issues in the months after birth, such as dyspareunia, lack of vaginal lubrication and a loss of sexual desire. For some women, these issues can persist 12 and 18 months after birth. Mode of birth is not associated with long-term dyspareunia 6 and 12 months after birth. There is conflict seen in the literature with regard to the association between perineal trauma and short-and long-term sexual health. Breastfeeding and the existence of pre-existing sexual health issues are strongly predictive of sexual health issues at 6 and 12 months after birth. Women have described a discordance in their sexual desire to that of their partner, for some this caused distress but for couples who communicated their feelings of sexual desire, concern over baby's well-being and adapting to parenthood distress was not experienced. Resuming sexual intercourse after birth was not spontaneous, women considered their mode of birth, the presence of perineal trauma and their physical and emotional recovery from birth. One fifth of women had not resumed sexual intercourse 12 weeks after birth.

Summary A discussion is presented on the challenges associated with viewing postpartum sexual health from a physical perspective only, and why prevalence studies alone do not capture the nuances of postpartum sexual health. Future research needs to take account of the psychosocial and relational dimensions of postpartum sexual health as well as physical dimensions.
\end{abstract}

\section{Introduction}

Research examining postpartum sexual health has increased dramatically over the past 20 years. Geraldine Barrett's cross-sectional study in 2000 was one of the first to explicitly ask a large number of women $(n=484)$ about their sexual health 6 months after the birth of their first baby [1]. Subsequent studies over the course of the next decade focused primarily on the physical aspects of postpartum sexual health, namely, dyspareunia, vaginal lubrication, orgasm, sexual arousal and timing of first sexual intercourse after birth, taking a one-dimensional

This article is part of the Topical Collection on Sociocultural Issues and Epidemiology

Deirdre O'Malley

omalled1@tcd.ie

1 School of Nursing and Midwifery, University of Dublin Trinity College, 24 D’Olier Street, Dublin DO2T283, Ireland view of postpartum sexual health [2-7]. These studies, however, were often underpowered, and retrospective in design with data collected early in the postpartum period (e.g. 3 to 6 months after birth) [7-11]. The last decade has seen a more comprehensive scrutiny of postpartum sexual health, incorporating physical $[12 \bullet, 13,14]$, social (adapting to parenthood, changed roles) [15, 16, 17•, 18], psychological (perception of body image, desire, fear, worry and anxiety) [19-21] and relational (emotional and practical support, perception of sexual desire in partner and changed roles) dimensions [22]. This paper presents a discussion on the recent evidence on postpartum sexual health. It begins with a review of the prevalence of postpartum sexual health issues and discusses the challenges associated with interpreting prevalence results. The possible risk factors associated with experiencing postpartum sexual health issues are then discussed. Women's views and experience of their sexual health after birth are also explored. 


\section{Prevalence of Postpartum Sexual Health Issues}

It is recognised that many women experience several sexual health issues after the birth of their first baby. Comprehensive accounts of the prevalence of these issues were recently published in two large scale prospective cohort studies carried out in Ireland [12•] and Australia [23]. In these studies, data were collected from postpartum women using similar survey instruments, which were administered at similar timepoints, thus enabling direct comparisons (Table 1).

Many prevalence studies on women's sexual health after birth report their results in terms of sexual disfunction and sexual problems. For example, in a comparison study of sexual function between nulliparous and multiparous women, authors report that sexual dysfunction was more frequent in nulliparous women [24]. A systematic review on women's experience of sex and intimacy in the first year postpartum included studies dating from 1996 to 2011 discussed findings in terms of prevalence of sexual problems [25]. Unlike these neither O'Malley and colleagues nor McDonald et al. suggest that $48-60 \%$ of women are 'sexually dysfunctional' because they experience a lack of vaginal lubrication or a loss of interest in sex [12•,23], rather their findings serve to highlight the fact that many women experience a number of different postpartum sexual health issues which persist for many months after the birth of their first baby. Frequently, in the earlier studies that examined women's postpartum sexual health, the Female Sexual Function Index (FSFI), a scale that measures sexual arousal, vaginal lubrication, achieving orgasm, dyspareunia and sexual satisfaction [26], was used, with lower scale scores used as markers of sexual dysfunction [23, 24]. Use of FSFI, however, is problematic for several reasons. The scale, which originated from the dated and disputed Master and Johnson Sexual Response Cycle [27], is now obsolete in terms of the DSM-V classifications of female sexual dysfunction as it does not take into account how long the woman is experiencing the issue and whether it is causing her distress [28]. We argue that it is questionable to label women as being sexually dysfunctional when they do not meet the criteria of the DSM-V classification for female sexual dysfunctions. Furthermore, the sexual response cycle described by Masters and Johnson propose that human sexual response cycle follows a linear model progressing through four phases: excitement, plateau, orgasm and resolution. It argues that sexual response is fundamentally identical in men and women. It lacks important components necessary for understanding female sexual response such as sexual desire, sexual motivation, emotional intimacy and relational dynamics. An intimacy-based model of female sexual response has been described by Basson (2000) (Fig. 1). This model redefined the phases of female sexual response and their relationship with one another. It suggests that with the experience of emotional intimacy, women may be receptive to sexual activity and may consequently

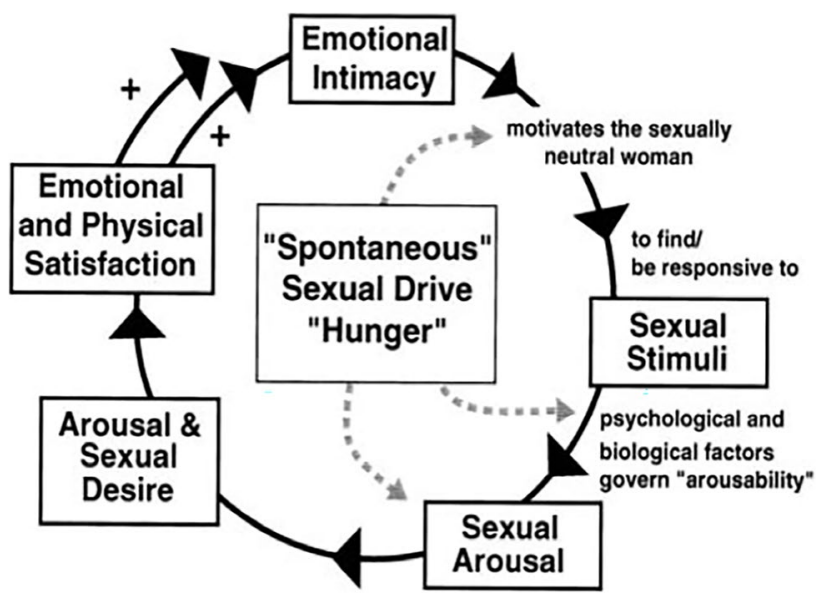

Fig. 1 An intimacy-based model of female sexual response (Basson 2001, p. 351)
Table 1 Prevalence of sexua health issues in nulliparous women from O'Malley et al. (2018) [12•] and McDonald et al. (2015) [23]

\begin{tabular}{llll}
\hline & & 6 months (\% n/total) & 12 months (\% n/total) \\
\hline Lack of vaginal lubrication & O'Malley et al. (2018) & $43 \%(283 / 685)$ & $35.4 \%(233 / 685)$ \\
& McDonald et al. (2015) & $48.2 \%(645 / 1349)$ & $36.9 \%(451 / 1138)$ \\
Pain during sexual intercourse & O'Malley et al. (2018) & $37.5 \%(236 / 629)$ & $20.5 \%(129 / 629)$ \\
& McDonald et al. (2015) & $43.4 \%(549 / 1349)$ & $29.5 \%(326 / 1138)$ \\
Difficulty reaching orgasm & O'Malley et al. (2018) & $30.7 \%(183 / 596)$ & $23.5 \%(140 / 596)$ \\
& McDonald et al. (2015) & $37.8 \%(502 / 1349)$ & $33.8 \%(380 / 1138)$ \\
Vaginal tightness & O'Malley et al. (2018) & $32.5 \%(200 / 616)$ & $17.4 \%(107 / 616)$ \\
& McDonald et al. (2015) & $34.1 \%(454 / 1349)$ & $20.8 \%(234 / 1138)$ \\
Vaginal looseness & O'Malley et al. (2018) & $13.4 \%(79 / 589)$ & $9 \%(53 / 589)$ \\
& McDonald et al. (2015) & $15.1 \%(199 / 1349)$ & $13.2 \%(147 / 1138)$ \\
Loss of interest in sex & O'Malley et al. (2018) & $46.3 \%(303 / 654)$ & $39.8 \%(260 / 654)$ \\
& McDonald et al. (2015) & $60.3 \%(814 / 1349)$ & $51.3 \%(581 / 1138)$ \\
\hline
\end{tabular}


experience arousal and desire, which can in turn lead to further desire for subjective and physiological sexual arousal, orgasm and feelings of emotional intimacy. In this model, subjective aspects of sexual arousal are emphasised in additional to physiological (genital and non-genital) responses [29-31].

The FSFI scale was not developed nor validated explicitly with postpartum women in mind, and thus its use in measuring sexual health issues in this discrete population, we argue, is questionable. For example, a study carried out in Poland administered a survey to 433 women between 10 and 52 weeks after birth in order to compare FSFI scores before and after birth [32]. Based on the results which showed that $44 \%$ of women scored significantly lower in their overall FSFI scoring after birth compared to before birth, the authors concluded that there was a 'strikingly' increase in sexual problems after birth. They also determined that $45.3 \%$ $(n=196)$ of women postpartum compared to $17.1 \%(n=74)$ pre-pregnancy had female sexual dysfunction [32]. Classifying women as being sexually dysfunctional on FSFI results alone ignores the complexity of postpartum sexual health and the potential influence of the wider context of women's lives and relationships, including the impact that adapting to parenting roles may have on intimate relationships. Additionally, the shorter timeframe of 10 weeks used in this study may be problematic, and potentially bias results, as it is likely that some women may not have resumed any form of sexual activity 10 weeks after birth. Nonetheless, FSFI scores continued to be significantly lower in women 52 weeks postpartum when compared to pre-birth scores; thus, authors described these women as being sexually dysfunctional.

Previous studies, conducted in Australia and Brazil, examining resumption of sexual activity and sexual intercourse after birth, found that a maximum of $78 \%$ of women attempted sexual intercourse by 12 weeks postpartum [33, 34]. Resumption rates ranged from $41 \%$ at 6 weeks [33], $51-65 \%$ at 8 weeks $[33,34]$ and $78 \%$ at 12 weeks after birth [33]. Hence, up to one-fifth of women may not have resumed any form of sexual activity 3 months after birth questioning the validity of labelling women as being sexually dysfunctional. In addition, many of the studies tend to frame and investigate the resumption of sexual activity from the lens of sexual intercourse taking no account of other non-coital activities, such as kissing, masturbation and oral sex. In summary, the use of the FSFI to measure women's postpartum sexual health is problematic for several reasons: it considers vaginal penetrative sexual intercourse only; the predominant emphasis is on physical measure of sexual response, that is, the presence or absence of sexual desire and arousal, dyspareunia, orgasm. The timing of collecting data early postpartum when women may not have resumed any form of sexual activity or when the data collection period is extended over months may bias results. Furthermore, it does not take into account the social, relational and psychological dimensions of postpartum sexual health. In recent years, a Spanish team developed and validated postpartum sexual health measurement tool; however, it too has a predominant emphasis on dyspareunia, sexual desire, arousal and a lack of vaginal lubrication [35]

This critique is not to detract from the evidence that many women experience sexual health issues after birth, and for some, these do cause distress; rather, it serves to highlight that using prevalence rates of physical sexual health measures alone as indicators of sexual dysfunction in postpartum women can be problematic. Adopting a more holistic approach that additionally encompasses emotional and social factors is likely required. For example, women reporting sexual concerns postpartum highlight issues such as when to resume sexual intercourse, frequency of sexual intercourse, tiredness, fear of dyspareunia and concern over perception of body image [36, 37]. Furthermore, psychosocial factors, such as tiredness, depression and relationship dissatisfaction, were associated with having moderate and marked sexual function problems [38•]. This indicates that sexual distress after birth for women is not necessarily related only to physical issues such as, dyspareunia, lack of vaginal lubrication and/or changed sexual desire but encompasses complex and multi-faceted factors that span the physical, emotional and social divides. Taking a more holistic view of postpartum sexual health does not inevitably results in increased prevalence rates rather is enables maternity healthcare providers to counsel and support women on possible changes to their sexual health after birth and to develop strategies and solutions to manage changes experienced.

\section{Risk Factors for Postpartum Sexual Health Issues}

Factors that influence women's postpartum sexual health are commonly explored and assessed in the literature. Frequently, an association between mode of birth and the development of postpartum dyspareunia is examined [12•, 13, 39-42]. In one study, of the 37\% $(n=73 / 193)$ of women who reported dyspareunia 10 to 14 months after birth, those who had an elective caesarean section were less likely to report dyspareunia in comparison to any other mode of birth group [42]. In another study, women who had an operative vaginal birth had a greater than threefold increase in dyspareunia 6 months postpartum; this may be explained by the likely associated episiotomy with operative vaginal births. Women who had an emergency caesarean section had a twofold increased odds of dyspareunia, compared to woman who had a spontaneous vaginal birth with an intact perineum when other maternal and obstetric factors were taken into account 
[13], although these associations did not persist 18 months after birth [39]. It is possible that the trauma associated with an emergency caesarean section and possible protracted labour not seen in an elective caesarean section might influence the association with postpartum dyspareunia. These results, which suggest a relationship between mode of birth and dyspareunia, are contradicted by O'Malley et al., where in a multivariate logistic regression analysis of potential risk factors for sexual health issues after birth, found that mode of birth was not a risk factor for postpartum dyspareunia, when normal vaginal birth was the reference category [12•]. Rather, they determined, in adjusting for the confounders of the following: age, BMI, level of education, mode of birth, degree of perineal trauma, pre-existing dyspareunia, breastfeeding and perception of body image that pre-pregnancy dyspareunia (adjusted odds ratio (AOR) 2.6, 95\% confidence interval (CI) 1.8 to 3.6) and breastfeeding (AOR 1.9, 95\% CI 1.3 to 2.7) were associated with dyspareunia 6 months postpartum. These associations persisted to 12 months after birth. The association with breastfeeding and dyspareunia is likely related to the association with breastfeeding and lack of vaginal lubrication (AOR 2., 95\% CI 1.5-2.9) and loss of interest in sex (AOR 2.2 95\% CI 1.6-2.9) seen in the same study.

The potential influence of perineal trauma, that is, tears, lacerations or incisions to the perineum during birth is of interest to many in this field of study. A systematic review by Korzeniewski et al. determined that perineal trauma was significantly associated with sexual difficulties (dyspareunia, lack of lubrication, unwanted leakage of urine or faeces during sexual intercourse, lack of sensation, vagina too tight or too lax) [25]. These conclusions, although based on the results from 13 studies $(n=5995)$, used various methodologies, and thus, the data could not be meta-analysed. There is a notable heterogeneity, in that samples included nulliparous and multiparous women, data were collected at varying and wide timeframes ( $24 \mathrm{~h}$ after birth to 12 months postpartum), many used non-validated data collection tools that often focused on physical attributes of postpartum sexual health only. Frequently, pre-existing sexual health issues were not accounted for in included studies. Furthermore, the quality of the included studies was not independently assessed by a second reviewer; thus, caution should be advised when considering the clinical applicability of these findings. Another systematic review (14 studies, $n=9095$ ) found that women who experienced severe perineal trauma $\left(3^{\text {rd }}\right.$ or $4^{\text {th }}$ degree perineal lacerations) were more predisposed to delaying resumption of sexual intercourse after birth and experiencing dyspareunia [43]. This systematic review has similar limitations to that of Korzeniewski et al.; whereby, although an extensive search of the databases was performed, the results are reported narratively rather than meta-analysing the data, with the absence of a quality appraisal of included studies.
Included studies had varied and wide-ranging data collection timepoints (up to 6 years), use multiple data collection tools and were reliant on women to self-identify as having severe perineal laceration. There was an assumption that delayed resumption of sexual intercourse was a result of the perineal trauma only and not associated with cultural preferences, extreme tiredness, adapting to parenthood or general and/ mental health problems. Although these reviews suggest an association between perineal trauma and sexual health issues postpartum, the strength of the evidence remains uncertain, with higher level evidence, based on an assessment of the quality of studies reporting these associations, and metaanalytical evidence required.

There are often conflicting results on the influence of perineal trauma on postpartum sexual health, issues relating to sample size, categorising perineal trauma and retrospective study designs complicate interpretation of results. However, there does appear to be an association between any form of perineal trauma and 'delaying' resuming sexual intercourse until 6 weeks postpartum. Women who had an episiotomy or a sutured perineal tear were less likely to have resumed sexual intercourse 6 weeks after birth (OR 2.13 CI 95\% 1.6-2.9 and OR 1.86 CI 95\% 1.4-2.4) respectively, compared to those who had an intact perineum [33]. This association persisted to 12 weeks after birth for women who had an episiotomy. Experiencing severe perineal trauma does appear to be predictive of delaying sexual intercourse to 3 months postpartum. In a Norwegian study, women who had severe perineal trauma $(n=28 / 533)$ were significantly more likely to report 'having difficulty with coitus' and delay resumption of sexual intercourse 2 and 3 months after birth when all other possible risk factors were included in the analysis [44]. The association between any form of perineal trauma and persistent sexual health issues was not seen by O'Malley et al. 6 and 12 months postpartum when all other potential risk factors were considered. This supports the notion that prevalence of sexual health issues decreases as postpartum months elapse. For example, Mc Donald and colleagues showed a reduction in the prevalence of dyspareunia, lack of vaginal lubrication, loss of interest in sex and problems with orgasm from 3 to 6 months and again from 6 to 12 months after birth [23]. Over time soft tissues heal, parents adapt to new roles and sleep becomes more regular, all potentially having a significant influence on the prevalence of sexual health issues. It is noteworthy that in studies using qualitative designs, women described how they waited until they had physically recovered from the birth when planning to resuming sexual activity. Women reported waiting for abdominal and perineal wounds to heal and lochia to cease in advance of resuming sexual activity [37, 45], which suggest that short-term physical recovery from birth is an important consideration for postpartum sexual health. Additionally, it is likely that some women were advised by 
their maternity healthcare provider to wait until 6 weeks had passed and they were assessed by their general practitioner or obstetrician before resuming sexual intercourse.

The maternal, infant and child health benefits of breastfeeding are well recognised [46]. However, discussions on the potential impact of breastfeeding on sexuality are often absent within studies, as well as absent from the wider discourse on breastfeeding. Physiologically during lactation oestrogen levels are low, which affects the epithelial lining of vaginal mucosa resulting in a thinning of the mucosa and a loss of lubrication, both associated with dyspareunia. Furthermore, increased prolactin levels (necessary for lactation) are known to impact sex hormones, including testosterone, which may have an influence on sexual desire, sexual arousal and orgasm. Not only is breastfeeding associated with a lack of vaginal lubrication, it has also been shown to increase the likelihood of experiencing persistent sexual health issues, namely, dyspareunia and loss of interest in sex, at 6 and 12 months after birth [12•]. In another study, breastfeeding women $(n=337)$ were 1.5 times more likely to experience dyspareunia 6 months after birth than their counterparts who were not breastfeeding $(n=373)$ [13]. Furthermore, women who were breastfeeding were less likely to report high rates of sexual pleasure then those who were not breastfeeding 6 months postpartum (AOR 0.62) [47]. In a Canadian study involving 239 new-parent couples of a healthy infant aged 3 to 12 months [36], mothers, and fathers to a lesser extent, reported 'concern' about the effect of breastfeeding on breasts and sexuality through completion of an online survey. This 'concern' may be explained though other research, where women described being 'uncomfortable' with the dual role of their breasts, that is, the role of the breast as a source of nutrition for their baby and the breasts as a source of sexual satisfaction [37].

\section{Women's Views of Postpartum Sexual Health}

There is an emerging trend of qualitative study designs being utilised to give voice to women's views and experiences of their postpartum sexual health $[15,37,45,48,49]$. A common theme from these studies is that of a discordance of sexual desire between women and their partners in the months following birth, which for many, causes feelings of guilt and failure that they are not living up to their own expectation of sexual activity postpartum $[15,37,45,50,51]$. Incorporated in this is women's awareness of their partner's sexual desire, with women reporting that they did not want to 'hurt their partner's feelings' by regularly refusing their partners sexual advances. In some cases, women managed this by engaging in sexual intercourse when they felt no desire [15, 37], with some pretending they felt sexual desire and faking an orgasm [50]. A Canadian study that explored how new parents ( $n=23$ couples, including 4 same sex relationship) experienced their intimate and sexual relationship as they adapted to parenthood [52] found that although couples were aware of a discordance in sexual desire, for most, it did not cause distress, with many reporting being satisfied with their sexual and intimate relationship. Couples accepted the changes to their relationship that parenthood brought, planning or scheduling sex and acknowledged a new depth and closeness to their relationship that was not their before becoming parents [52]. This study is unique in that the sample includes four couples in same-sex relationship, voices that are absent in the majority of studies on postpartum sexual health.

An interpretative phenomenological study with eight women, 6 and 12 months postpartum, described women's need for physical and emotional intimacy rather than sexual intimacy [51]. In this study, physical intimacy referred to holding hands, a kiss, a cuddle or a back rub; emotional intimacy represented trust, respect, understanding and good communication within the couple dyad. Similar views were described by women in a qualitative descriptive study utilising individual semi-structured interviews with twenty-one nulliparous women [37]. In this study, when women talked about their desire to maintain intimacy with their partner in the months after birth, intimacy was not sexual activity per se rather it encompassed sitting together on the couch, watching a TV show together and regular kisses and cuddles. It was also evident that a significant feature of maintaining a satisfying and intimate relationship in the postpartum months was communication about discordance in sexual desire, feelings of guilt associated with this, communication about baby care and adapting to new roles [37]. Further evidence of the association between effective communication and satisfying intimate relationships over time (6 months, 4 and 8 years after the birth of the first baby) has been demonstrated by Tone Ahlborg and colleagues in a longitudinal study involving 820 first time parents in Sweden [18, 53, 54].

Fatigue, tiredness and adapting to parenthood have featured extensively in the literature describing women's experience of postpartum sexual health. In a qualitative descriptive study, eighteen women, 2.5-3.5 years after the birth of their first baby, were interviewed about their experience of changes to their sexual relationships, sexual expression and intimacy as a result of childbirth and parenting [15]. The factors that emerged were, extreme tiredness, changed lifestyle, emotional connection to the baby, changes to the sexual and intimate relationship and changed view of sexuality. This corresponds with findings from a phenomenological study involving ten postpartum women who described how their new reality at home impacted on their sexual health [45]. Women talked about the changed family roles, sharing responsibility for childcare and the shift in relationship priorities; some women feared losing their womanhood as 
baby care took priority. Women have expressed a need for personal space away from the baby and their partner in order to rest, sleep or read a book or magazine [37, 45].

Women's perception of their body image has featured as a theme when considering postpartum sexual health. While body image is an issue that impacts many women, postpartum body image is also impacted by scars, changed abdomen shape and size, enlarged breasts, stretch marks and changing body weight $[37,45,51,55]$. A prospective cohort study in Ireland $(n=832)$ demonstrated that women who were 'never satisfied' with their body image were more likely to selfreport a loss of interest in sex at 6 and 12 month postpartum (AOR 2.8 and 3.6, respectively) [12•]. The theme of negative perception of body image was often described in terms of women's loss of sexual identity, loss of sexual desire, infrequent and less satisfying sexual activity and physical changes to their body that women disliked and could not control $[15,51,56]$. Although a negative perception of body image is not always the case, a small number of women in the studies described an increase in self-esteem associated with their body's accomplishments, that is, growing and feeding a baby [37], and some enjoyed their larger breasts [51]. An association between body satisfaction, body image and sexual health was reported in a US study involving 168 nulliparous women [57]. Using a variety of validated scales to measure sexual function, body image self-consciousness, body satisfaction and genital self-image (women's feelings and beliefs about their own genitals), the authors of the study concluded that body image self-consciousness and genital self-image are predictors of postpartum sexual health. This is significant as other researchers have determined that women who experience perineal trauma tend to fare worse in terms of perception of body image, for example, women $(n=69)$ who had an episiotomy had significantly lower sexual/body esteem compared to those who did not 8 months postpartum [58].

In summary, this review has examined postpartum sexual health from multiple dimensions, including physical, psychosocial and relational. We consider that the framing of postpartum sexual health within a coital imperative and the absence of the voices of women who identify with a sexual orientation other than heterosexual within the literature is noteworthy and problematic. Sexuality is individual, expressed in all that we are. Postpartum sexual health, however, as a discrete part of sexuality is complex and multifaceted. Postpartum sexual health issues, we argue, cannot be reduced to objective physical measures; rather, to truly understand the sexual issues that women might experience postpartum, a holistic cognisance of social and emotional factors, in addition to physical outcome measures, is required. Future studies ought to examine the relationship between women's expectations of sex after birth, adapting to parenthood, extreme tiredness, the influence of stress and anxiety, communication within the couple dyad, division of household chores and baby care, non-sexual touch and sexual health after birth. Supporting women attain good postpartum sexual health requires an understanding of all potential influencing factors, which should be discussed with women in optimising the postpartum, and future, health and well-being of women and their families.

Funding Open Access funding provided by the IReL Consortium.

\section{Declarations}

Conflict of Interest The authors declare no competing interests.

Open Access This article is licensed under a Creative Commons Attribution 4.0 International License, which permits use, sharing, adaptation, distribution and reproduction in any medium or format, as long as you give appropriate credit to the original author(s) and the source, provide a link to the Creative Commons licence, and indicate if changes were made. The images or other third party material in this article are included in the article's Creative Commons licence, unless indicated otherwise in a credit line to the material. If material is not included in the article's Creative Commons licence and your intended use is not permitted by statutory regulation or exceeds the permitted use, you will need to obtain permission directly from the copyright holder. To view a copy of this licence, visit http://creativecommons.org/licenses/by/4.0/.

\section{References}

Papers of particular interest, published recently, have been highlighted as: • Of importance

1. Barrett G, et al. Women's sexual health after childbirth. Br J Obstet Gynaecol. 2000;107(2):186-95.

2. Buhling KJ, et al. Rate of dyspareunia after delivery in primiparae according to mode of delivery. Eur J Obstet Gynecol Reprod Biol. 2006;124(1):42-6.

3. Signorello LB, et al. Postpartum sexual functioning and its relationship to perineal trauma: a retrospective cohort study of primiparous women. Am J Obstet Gynecol. 2001;184(5):881-8; discussion 888-90.

4. Safarinejad MR, Kolahi AA, Hosseini L. The effect of the mode of delivery on the quality of life, sexual function, and sexual satisfaction in primiparous women and their husbands. J Sex Med. 2009;6(6):1645-67.

5. Klein K, et al. Does the mode of delivery influence sexual function after childbirth? J Womens Health. 2009;18(8):1227-31.

6. Dean N, et al. Sexual function, delivery mode history, pelvic floor muscle exercises and incontinence: a cross-sectional study six years post-partum. Aust N Z J Obstet Gynaecol. 2008;48(3):302-11.

7. Baksu B, et al. The effect of mode of delivery on postpartum sexual functioning in primiparous women. Int Urogynecol J Pelvic Floor Dysfunct. 2007;18(4):401-6.

8. DeJudicibus MA, McCabe MP. Psychological factors and the sexuality of pregnant and postpartum women. J Sex Res. 2002;39(2):94-103. 
9. Connolly A, Thorp J, Pahel L. Effects of pregnancy and childbirth on postpartum sexual function: a longitudinal prospective study. Int Urogynecol J Pelvic Floor Dysfunct. 2005;16(4):263-7.

10. Leeman LMMD, Rogers RGMD. Sex after childbirth: postpartum sexual function. Obstet Gynecol. 2012;119(3):647-55.

11. Hosseini L, Iran-Pour E, Safarinejad MR. Sexual function of primiparous women after elective cesarean section and normal vaginal delivery. Urol J. 2012;9(2):498-504.

12. O'Malley D, et al. Prevalence of and risk factors associated with sexual health issues in primiparous women at 6 and 12 months postpartum; a longitudinal prospective cohort study (the MAMMI study). BMC Pregnancy Childbirth. 2018;18(1):196. This is a large cohort study with nulliparous women in Ireland. Data were collected at five timepoint; antenatally, 3, 6, 9 and 12 months postpartum. The large sample size and prospective design enabled a true picture of prevalence of sexual health issues during the first 12 months after birth. Furthermore, univariate and multivariate analyses allowed for the identification of risk factors for long-term sexual health issues.

13. McDonald EA, et al. Dyspareunia and childbirth: a prospective cohort study. BJOG Int J Obstet Gynaecol. 2015;122(5):672-9.

14. Bertozzi S, et al. Prevalence and risk factors for dyspareunia and unsatisfying sexual relationships in a cohort of primiparous and secondiparous women after 12 months postpartum. Int J Sex Health. 2010;22(1):47-53.

15. Woolhouse H, McDonald E, Brown S. Women's experiences of sex and intimacy after childbirth: making the adjustment to motherhood. J Psychosom Obstet Gynecol. 2012;33(4):185-90.

16. Woolhouse H, McDonald E, Brown SJ. Changes to sexual and intimate relationships in the postnatal period: women's experiences with health professionals. Aust J Prim Health. 2014;20(3):298-304.

17. O'Malley D, Smith V, Higgins A. Women's solutioning and strategising in relation to their postpartum sexual health: a qualitative study. Midwifery. 2019. This is the only study we are aware of that identified how women manage their sexual health after the birth of their first baby. This study demonstrated that there was a silence from healthcare professionals on the topic and many sourced solutions themselves online and from female friends. Results demonstrated that women wanted to be prepared for changes to their intimate relationship. Furthermore, it identified that communication within the couple dyad was essential to adapting to the new sexual norm in the relationship.

18. Hansson M, Ahlborg T. Quality of the intimate and sexual relationship in first-time parents - A longitudinal study. Sex Reprod Healthc. 2012;3(1):21-9.

19. Lindqvist M, et al. "Struggling to settle with a damaged body" - A Swedish qualitative study of women's experiences one year after obstetric anal sphincter muscle injury (OASIS) at childbirth. Sex Reprod Healthc. 2019;19:36-41.

20. Mickelson KD, Joseph JA. Postpartum body satisfaction and intimacy in first-time parents. Sex Roles. 2012;67(5-6):300-10.

21. Moel JE, et al. Sexual function in the postpartum period: effects of maternal depression and interpersonal psychotherapy treatment. Arch Womens Ment Health. 2010;13(6):495-504.

22. O'Malley D, Higgins A, Smith V. Postpartum sexual health: a principle-based concept analysis. J Adv Nurs. 2015;71(10):2247-57.

23. McDonald E, Woolhouse H, Brown S. Consultation about sexual health issues in the year after childbirth: a cohort study. Birth. 2015;42(4):354-61.
24. Banaei M, et al. A comparison of sexual function in primiparous and multiparous women. J Obstet Gynaecol. 2020;40(3):411-8.

25 Korzeniewski R, Kiemle G, Slade P. Mothers' experiences of sex and sexual intimacy in the first postnatal year: a systematic review. Sex Relatsh Ther. 2019;36:1-19.

26. Rosen R, Brown C, Heiman J. The female sexual function index (FSFI): a multidimensional self-report instrument for the assessment of female sexual function. J Sex Marital Ther. 2000;26.

27. Masters W, Johnson V. Human Sexual Response. New York: Little Brown; 1966.

28. Diagnostic and statistical manual of mental disorders, 5th edition, text revision. 2013, Washington, DC: American Psychiatric Press.

29. Basson R. Are our definitions of women's desire, arousal and sexual pain disorders too broad and our definition of orgasmic disorder too narrow? J Sex Marital Ther. 2002;28(4):289-300.

30. Basson R. The female sexual response: a different model. J Sex Marital Ther. 2000;26(1):51-65.

31. Basson R. Recent advances in women's sexual function and dysfunction. Menopause. 2004;11(6):714-25.

32. Zgliczynska M, et al. Impact of childbirth on women's sexuality in the first year after the delivery. J Obstet Gynaecol Res. 2021;47(3):882-92.

33. McDonald EA, Brown SJ. Does method of birth make a difference to when women resume sex after childbirth? BJOG Int J Obstet Gynaecol. 2013;120(7):823-30.

34. Faisal-Cury A, et al. The relationship between mode of delivery and sexual health outcomes after childbirth. J Sex Med. 2015;12(5):1212-20.

35. López-Lapeyrere $\mathrm{C}$, et al. The development and validation of a new postpartum sexual function and dyspareunia assessment tool: The Carol Scale. Midwifery. 2018;58:27-36.

36. Schlagintweit HE, Bailey K, Rosen NO. A new baby in the bedroom: frequency and severity of postpartum sexual concerns and their associations with relationship satisfaction in new parent couples. J Sex Med. 2016;13(10):1455-65.

37. O'Malley D, Smith V, Higgins A. Women's solutioning and strategising in relation to their postpartum sexual health: a qualitative study. Midwifery. 2019;77:53-9.

38. Dawson SJ, et al. Biopsychosocial predictors of trajectories of postpartum sexual function in first-time mothers. Health Psychol. 2020;39(8):700-10. This study examines the relationship between biopsychosocial factors and postpartum sexual health. While it does utilise the FSFI, they also measured depression, fatigue, relationship satisfaction and sexual distress (that is feelings of distress around sex). Adjustments were made for women who were not sexually active in the preceding four; therefore, results were not skewed in the FSFI. The sample included $8 \%(n=48)$ who did not identify as being in a same sex relationship. The results showed that improvement in sexual function postpartum is heterogeneous. Psychosocial, but not biomedical factors were significantly associated with the three sexual health trajectories identified, that is, $52 \%$ of women reported minimal sexual function problems at 3 months postpartum and improved the least over time, $35 \%$ of women reported moderate sexual function problems at 3 months and improved the most over time and $13 \%$ of women reported marked sexual function problems at 3 months and improved somewhat over time.

39. McDonald E, et al. Frequency, severity and persistence of postnatal dyspareunia to 18 months post partum: a cohort study. Midwifery. 2016;34:15-20.

40. Triviño-Juárez J-M, et al. Resumption of intercourse, selfreported decline in sexual intercourse and dyspareunia in women by mode of birth: a prospective follow-up study. J Adv Nurs. 2018;74(3):637-50. 
41. Novo R, et al. Prevalence and associated risk factors of urinary incontinence and dyspareunia during pregnancy and after delivery. Eur J Obstet Gynecol Reprod Biol. 2020;245:45-50.

42. Lipschuetz M, et al. Degree of bother from pelvic floor dysfunction in women one year after first delivery. Eur J Obstet Gynecol Reprod Biol. 2015;191:90-4.

43. Andreucci CB, et al. Sexual life and dysfunction after maternal morbidity: a systematic review. BMC Pregnancy Childbirth. 2015;15(1):307.

44. Fodstad K, Staff AC, Laine K. Sexual activity and dyspareunia the first year postpartum in relation to degree of perineal trauma. Int Urogynecol J Pelvic Floor Dysfunct. 2016;27(10):1513-23.

45. Pardell-Dominguez L, et al. The meaning of postpartum sexual health for women living in Spain: a phenomenological inquiry. BMC Pregnancy Childbirth. 2021;21(1):1-13.

46. World Health Organization. Breastfeeding. 2021 [cited 2021 28th Jun 2021]; Available from: https://www.who.int/healthtopics/breastfeeding\#tab=tab_1.

47. McDonald E, Woolhouse H, Brown SJ. Sexual pleasure and emotional satisfaction in the first 18 months after childbirth. Midwifery. 2017;55:60-6.

48 Rosen NO, Bailey K, Muise A. Degree and direction of sexual desire discrepancy are linked to sexual and relationship satisfaction in couples transitioning to parenthood. J Sex Res. 2017;55:1-12.

49 Hipp LE, Kane Low L, van Anders SM. Exploring women's postpartum sexuality: social, psychological, relational, and birthrelated contextual factors. J Sex Med. 2012;9(9):2330-41.

50. Olsson A, et al. Women's thoughts about sexual life after childbirth: focus group discussions with women after childbirth. Scand J Caring Sci. 2005;19(4):381-7.

51 Bender SS, Sveinsdóttir E, Fridfinnsdóttir H. "You stop thinking about yourself as a woman". An interpretive phenomenological study of the meaning of sexuality for Icelandic women during pregnancy and after birth. Midwifery. 2018;62:14-9.

52. Lévesque $S$, et al. A study of the transition to parenthood: new parents' perspectives on their sexual intimacy during the perinatal period. Sex Relatsh Ther. 2019;36:1-18.

53. Ahlborg T, Strandmark M. Factors influencing the quality of intimate relationships six months after delivery-first-time parents' own views and coping strategies. J Psychosom Obstet Gynecol. 2006;27(3):163-72.

54. Ahlborg T, Misvaer N, Möller A. Perception of marital quality by parents with small children: a follow-up study when the firstborn is 4 years old. J Fam Nurs. 2009;15(2):237-63.

55. Martínez-Martínez A, et al. The sexual function of women during puerperium: a qualitative study. Sex Relatsh Ther. 2017;32(2):181-94.

56. Khajehei M, Doherty M. Women's experience of their sexual function during pregnancy and after childbirth: a qualitative survey. Br J Midwifery. 2018;26(5):318-28.

57. Jawed-Wessel S, Herbenick D, Schick V. The relationship between body image, female genital self-image, and sexual function among first-time mothers. J Sex Marital Ther. 2017;43(7):618-32.

58. Zielinski R, et al. Body after baby: a pilot survey of genital body image and sexual esteem following vaginal birth. Int J Women's Health. 2017;9:189-98.

Publisher's Note Springer Nature remains neutral with regard to jurisdictional claims in published maps and institutional affiliations. 